\title{
High resolution mesospheric sodium properties for adaptive optics applications ${ }^{\star}$
}

\author{
T. Pfrommer ${ }^{1}$ and P. Hickson ${ }^{1,2,3}$ \\ ${ }^{1}$ European Southern Observatory (ESO), Karl-Schwarzschild-Strasse 2, 85758 Garching bei München, Germany \\ e-mail: tpfromme@eso.org \\ 2 University of British Columbia, Department of Physics and Astronomy, 6224 Agricultural Road, Vancouver, BC, V6T1Z1, Canada \\ e-mail: hickson@phas.ubc.ca \\ ${ }^{3}$ Institut d'Astrophysique et de Géophysique, Université de Liège, Allée du 6 août, 17 - Bat. B5c, 4000 Liège 1, Belgique
}

Received 19 January 2014 / Accepted 20 March 2014

\section{ABSTRACT}

\begin{abstract}
Context. The performance of laser guide star adaptive optics (AO) systems for large optical and infrared telescopes is affected by variability of the sodium layer, located at altitudes between 80 and $120 \mathrm{~km}$ in the upper mesosphere and lower thermosphere. The abundance and density structure of the atomic sodium found in this region is subject to local and global weather effects, planetary and gravity waves and magnetic storms, and is variable on time scales down to tens of milliseconds, a range relevant to AO.

Aims. It is therefore important to characterize the structure and dynamical evolution of the sodium region on small, as well as large spatial and temporal scales. Parameters of particular importance for AO are the mean sodium altitude, sodium layer width and the temporal power spectrum of the centroid altitude.

Methods. We have conducted a three-year campaign employing a high-resolution lidar system installed on the 6-m Large Zenith Telescope (LZT) located near Vancouver, Canada. During this period, 112 nights of useful data were obtained.

Results. The vertical density profile of atomic sodium shows remarkable structure and variability. Smooth Gaussian-shaped profiles rarely occur. Multiple internal layers are frequently found. These layers often have sharp lower edges, with scale heights of just a few hundred meters, and tend to drift downwards at a typical rate of one kilometer every two to three hours. Individual layers can persist for many hours, but their density and internal structure can be highly variable. Sporadic layers are seen reaching peak densities several times the average, often in just a few minutes. Coherent vertical oscillations are often found, typically extending over tens of kilometers in altitude. Regions of turbulence are evident and Kelvin-Helmholtz instability are sometimes seen. The mean value of the centroid altitude is found to be $90.8 \pm 0.1 \mathrm{~km}$. The sodium layer width was determined by computing the altitude range that contains a specified fraction of the returned sodium light. We find a mean value of $13.1 \pm 0.3 \mathrm{~km}$ for the range containing $95 \%$ of the photons, with a maximum width of $21 \mathrm{~km}$. The temporal power spectral density of fluctuations of the centroid altitude is well described by a power law having an index that ranges from -1.6 to -2.3 with a mean value of $-1.87 \pm 0.02$. This is significantly steeper than the value of $-5 / 3$ that would be expected if the dynamics were dominated by Kolmogorov turbulence, indicating that other factors such as gravity waves play an important role. The amplitude of the power spectrum has a mean value of $34_{-5}^{+6} \mathrm{~m}^{2} \mathrm{~Hz}^{-1}$ at a frequency of $1 \mathrm{~Hz}$, but ranges over two orders of magnitude. The annual means of the index and amplitude show a variation that is well beyond the calculated error range. Long-term global weather patterns may be responsible for this effect.
\end{abstract}

Key words. atmospheric effects - instrumentation: adaptive optics - site testing - methods: observational

\section{Introduction}

Next-generation extremely-large ground-based telescopes (ELTs) will provide unprecedented sensitivity and resolution, enabling astronomers to address many open scientific topics ranging from the formation and evolution of galaxies to the characterization of exoplanets (Lyubenova \& Kissler-Patig 2009; Silva et al. 2007). These telescopes will achieve high resolution by mean of adaptive optics (AO) systems (Beckers 1993). AO systems employ wavefront sensors (WFS) to measure the wavefront distortion introduced by atmospheric turbulence along the line of sight to reference "guide" stars. A correction is then computed in real time and the wavefront is corrected by means of deformable mirrors (Ellerbroek et al. 2008). To match atmospheric time scales, information about the instantaneous wavefront error (WFE) requires bright guide stars. This restricts

\footnotetext{
* The database is only available at the CDS via anonymous ftp to cdsarc.u-strasbg. fr (130.79.128.5) or via http://cdsarc.u-strasbg.fr/viz-bin/qcat?J/A+A/565/A102
}

the use of $\mathrm{AO}$ to a small sky coverage because sufficiently bright natural guide stars are rare (Wang et al. 2009). Thus, laser systems are employed to create artificial guide stars, either by Rayleigh scattering in the lower $20 \mathrm{~km}$ of the atmosphere or by resonant excitation of sodium atoms in the upper mesosphere and lower thermosphere. Rayleigh systems are well-suited for smaller telescopes and ground-layer AO systems. However, with large apertures, differences in propagation paths between light from the guide star and the science target (the cone effect) is more severe due to the low altitude of the Rayleigh guide star. Therefore sodium laser guide stars are preferred for ELTs.

These laser guide stars (LGS) appear as luminous columns of backscattered light in the $\sim 10-20 \mathrm{~km}$ thick sodium region. They appear star-like when seen from directly below, but elongated when seen from regions of the entrance pupil that are offset from the laser, an effect that increases with telescope aperture. This elongation spreads the sodium light over more WFS pixels, reducing the signal-to-noise ratio. Furthermore, the intensity structure within the elongated LGS is irregular and variable, 
reflecting the evolving density structure of the sodium region. This introduces an uncertainty in the location of the centroid of the LGS image, degrading the performance of the AO system even more. This error increases linearly with LGS elongation, and is thus proportional to the telescope diameter.

Variations in the altitude of the centroid of the vertical density distribution of the atomic sodium results in shifts in the position of the centroid of the LGS. The AO system interprets this as a change in focus, but cannot distinguish it from focus variations that are produced by atmospheric turbulence (which need to be corrected in order to keep the science target in focus). Natural guide stars (NGS) can be used to break the degeneracy, but only at low temporal frequencies (Herriot et al. 2006). At a given temporal frequency, the resulting focus wavefront error is proportional to the square of the telescope diameter. However, larger apertures collect more NGS photons allowing more frequent measurement of the focus, which partially offsets this effect. The net result depends on the spectral index of the sodium centroid altitude power spectrum.

Atmospheric lidars have been employed to study the structure of the sodium region for several decades (Clemesha et al. 1980; Gardner et al. 1986; von Zahn \& Hansen 1988; She et al. 1991). These systems generally have small apertures and require integration times of several minutes in order to achieve sufficient signal-to-noise ratios. However, AO systems are sensitive to frequencies as high as a $\mathrm{kHz}$. Performance estimates for AO systems on ELTs have thus relied on a highly uncertain extrapolation, over four decades of frequency, from existing lowfrequency measurements (Davis et al. 2006). This circumstance motivated us to design a sodium lidar (light detection and ranging) system for the Large Zenith Telescope (LZT), located near Vancouver, Canada at $49^{\circ} \mathrm{N}$ latitude (Hickson et al. 2007). The 6-m aperture of this telescope provides an increase of as much as two orders of magnitude in light gathering power compared to typical lidars. It is well-suited to studies of mesospheric sodium on timescales relevant for AO. A sodium layer study for LGS AO purposes on time resolutions of tens of seconds has been conducted by Neichel et al. (2013) on Cerro Pachón in the southern hemisphere. Their dynamical results agree well with the present 3-year data set.

This paper briefly describes the lidar system and presents the results of a three-year observing campaign.

\section{Observations}

A general description of the lidar system can be found in Pfrommer et al. (2008) and a detailed description, including all subsystems, is given in Appendix A of Pfrommer (2010). The lidar employs a $13 \mathrm{~W} \mathrm{Nd-YAG} \mathrm{frequency-doubled} \mathrm{pulsed} \mathrm{laser}$ emitting $532 \mathrm{~nm}$ radiation, which pumps a dye laser tuned to $589 \mathrm{~nm}$. The delivered average output power is $4-5 \mathrm{~W}$. The pulse repetition frequency is $50 \mathrm{~Hz}$ and the pulse width is $\sim 6 \mathrm{~ns}$. A $15 \mathrm{~cm}$ refracting telescope expands and collimates the beam, and a 45 degree flat mirror directs it vertically. Backscattered light is collected by the f/1.5 LZT primary mirror and directed to the lidar receiver located at the prime focus. A rotating chopper wheel, synchronized with the laser trigger, blocks Rayleighscattered light from the atmosphere, but admits light returning from the sodium region. This light passes through a 1-arcmin diameter field stop and is directed to a segmented collimator mirror. The four segments of this concave mirror are tilted slightly so as to split the light and multiplex it to four parallel receiver channels. This improves sensitivity by reducing coincidence losses and also adds redundancy. Each channel contains refocusing optics, a narrow-band filter, and a high-efficiency GaAsP-based photomultiplier. Counts from the four photomultipliers are discriminated, timed and accumulated in a high-speed 4-channel pulse-counting system.

This system provides high resolution Na-profiles with $4 \mathrm{~m}$ height and $20 \mathrm{~ms}$ time resolution, allowing us to study, for the first time, sodium layer dynamics on scales important to AO systems (Pfrommer et al. 2009). Typically about $10^{3}$ returned photons are detected for each laser pulse. This results in an uncertainty in the centroid altitude on the order of $\sim 50$ to $100 \mathrm{~m}$. Averaging over one second (50 laser pulses) reduces this uncertainty by a factor of 7 . The resulting sodium profiles are being used to verify control algorithms and optimize their performance (Conan et al. 2009). In addition, our profiles are being used to improve return flux simulations for existing and new laser systems for LGS AO systems (Holzlöhner et al. 2010).

Over a three-year time span from 2008 to $2010,476 \mathrm{~h}$ of data were obtained, covering all seasons. After an initial commissioning phase, regular observations commenced in July 2008 using a "first-generation" photon-counting system. We obtained $110 \mathrm{~h}$ of observations in 2008 and a further $56 \mathrm{~h}$ up to the end of July 2009. In the fall of 2008, a failure of the optical encoder inside the LZT primary mirror air bearing necessitated a shutdown for most of the winter. Beginning in August 2009 with engineering nights, a "second-generation" counting system was installed and regular observations began with this new and improved system in September 2009. $56 \mathrm{~h}$ of observations were obtained in the rest of 2009 and $256 \mathrm{~h}$ of observations were acquired in 2010. A summary of hours of observation obtained each month is given in Table 1.

\section{Analysis and results}

\subsection{Sodium layer structure}

In previous publications (Pfrommer et al. 2009; Pfrommer \& Hickson 2010) we reported on data obtained using the firstgeneration counting system. Qualitatively, we see the same kinds of structure with the new system, but at higher resolution and with no system-related data acquisition stops. The sodium region is generally resolved into multiple components which are horizontally stratified, forming layers which often overlap. These layers often have sharply-defined upper or lower boundaries.

Figure 1 shows an example of a sodium density map recorded on September 2009 with the new system. The three distinct black bars indicate system problems that were resolved during the night. The sodium structure in this figure is resolved with a vertical bin size of $75 \mathrm{~m}$ and temporal bin size of $2.5 \mathrm{~s}$. The ordinate represents the absolute altitude above sea level and the abscissa is the UT time. The color code indicates the number of photons per bin. Because the sodium layer is optically thin, the returned photon flux $F(z)$ from altitude $z$ is proportional to the number density $\rho(z)$ of sodium atoms,

$F(z) \propto \frac{\rho(z)}{h^{2}}$

where $h=z-z_{0}$ is the height above the lidar system, located at altitude $z_{0}$. In this figure, and for all subsequent results, we use photon flux as a proxy for sodium density, ignoring the geometric dilution factor $h^{-2}$. The reason for this is that our primary aim is to study the impact of sodium variability on AO systems. These systems do not correct for the geometric range (and do not need to), so our results are directly applicable to AO system modeling. In considering the physical processes affecting 
T. Pfrommer and P. Hickson: High resolution mesospheric sodium properties for adaptive optics applications

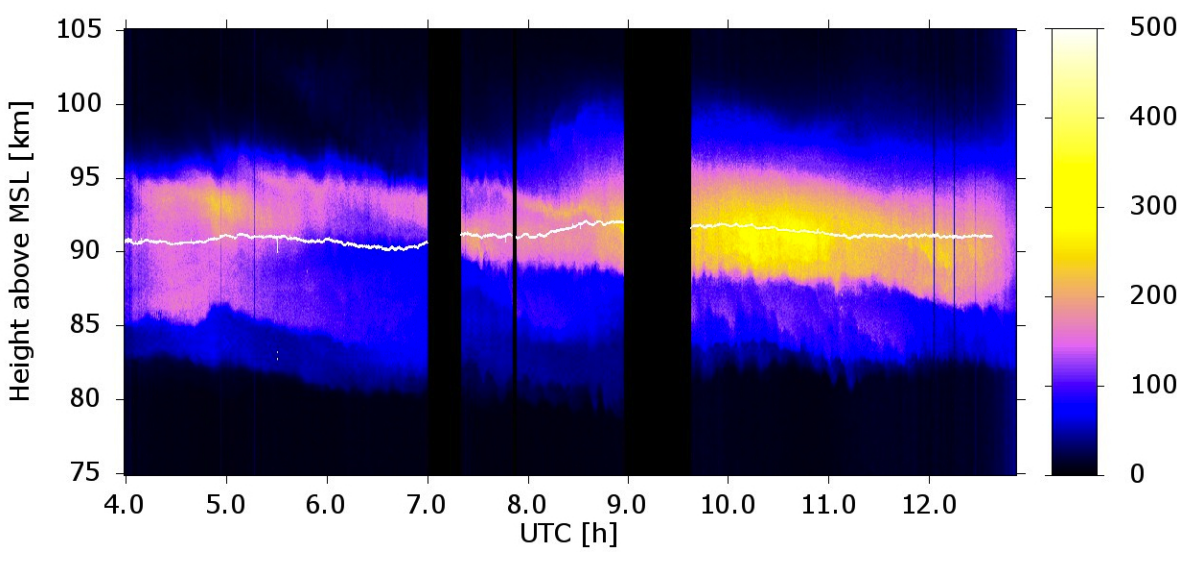

500

400

300

200

Fig. 1. Sodium density evolution for a typical night (Sept. 12th, 2009) is shown. The colour-coded intensity shows photon counts per bin. The white line indicates the flux-weighted mean altitude.

Table 1. Log of observations.

\begin{tabular}{|c|c|c|c|c|c|c|c|c|c|c|c|}
\hline Date & Start & End & Hours & Date & Start & End & Hours & Date & Start & End & Hours \\
\hline $2008 / 07 / 09$ & $8: 58$ & $10: 59$ & 2.0 & $2008 / 07 / 10$ & $5: 57$ & $10: 25$ & 4.5 & $2008 / 07 / 11$ & $5: 37$ & $10: 35$ & 4.6 \\
\hline $2008 / 07 / 12$ & $5: 43$ & $10: 44$ & 5.0 & $2008 / 07 / 13$ & $6: 52$ & $10: 47$ & 3.9 & $2008 / 07 / 15$ & 6:08 & $10: 35$ & 4.5 \\
\hline $2008 / 07 / 16$ & $6: 29$ & $7: 53$ & 1.4 & $2008 / 07 / 17$ & $6: 33$ & 11:01 & 4.5 & $2008 / 07 / 18$ & $5: 38$ & $10: 24$ & 4.8 \\
\hline $2008 / 07 / 19$ & $6: 58$ & $10: 04$ & 3.1 & $2008 / 07 / 22$ & $5: 44$ & $10: 45$ & 5.0 & $2008 / 07 / 23$ & $5: 40$ & 9:01 & 3.3 \\
\hline $2008 / 07 / 25$ & $5: 45$ & $11: 20$ & 5.5 & 2008/08/04 & $5: 46$ & $10: 55$ & 5.2 & $2008 / 08 / 05$ & $5: 50$ & $11: 12$ & 5.3 \\
\hline $2008 / 08 / 06$ & $5: 24$ & $11: 26$ & 6.0 & $2008 / 08 / 07$ & $4: 46$ & $5: 18$ & 0.3 & $2008 / 08 / 08$ & $4: 48$ & $8: 25$ & 3.6 \\
\hline $2008 / 08 / 15$ & 4:59 & $8: 15$ & 3.3 & $2008 / 09 / 30$ & $4: 15$ & $12: 05$ & 7.9 & $2008 / 10 / 10$ & $5: 55$ & $13: 21$ & 7.2 \\
\hline $2008 / 10 / 11$ & 4:09 & $13: 22$ & 9.2 & $2008 / 10 / 19$ & 7:05 & $10: 10$ & 3.1 & $2008 / 10 / 28$ & $1: 37$ & $11: 21$ & 9.9 \\
\hline $2008 / 10 / 29$ & $1: 08$ & $4: 06$ & 3.0 & $2009 / 06 / 29$ & $4: 24$ & $6: 24$ & 2.0 & $2009 / 06 / 30$ & $4: 18$ & $6: 15$ & 2.0 \\
\hline $2009 / 07 / 01$ & $4: 12$ & $6: 16$ & 2.0 & $2009 / 07 / 02$ & $6: 03$ & $6: 24$ & 0.3 & $2009 / 07 / 03$ & 4:07 & $7: 14$ & 3.1 \\
\hline $2009 / 07 / 04$ & 4:00 & $8: 31$ & 4.5 & $2009 / 07 / 11$ & $3: 34$ & $8: 25$ & 4.8 & $2009 / 07 / 12$ & $3: 21$ & $4: 45$ & 1.4 \\
\hline $2009 / 07 / 17$ & $3: 46$ & $5: 48$ & 2.0 & $2009 / 07 / 18$ & $3: 59$ & $8: 23$ & 4.4 & $2009 / 07 / 19$ & $3: 50$ & $8: 33$ & 4.7 \\
\hline $2009 / 07 / 20$ & $3: 01$ & $8: 05$ & 5.0 & $2009 / 07 / 21$ & 4:03 & $8: 33$ & 4.5 & $2009 / 07 / 22$ & $3: 24$ & $8: 30$ & 5.1 \\
\hline $2009 / 07 / 23$ & $3: 19$ & $8: 37$ & 5.3 & $2009 / 07 / 25$ & 4:59 & $6: 07$ & 1.1 & $2009 / 07 / 26$ & $3: 27$ & $8: 28$ & 5.0 \\
\hline $2009 / 07 / 27$ & $3: 03$ & $8: 15$ & 5.1 & $2009 / 07 / 28$ & $3: 05$ & $8: 16$ & 5.1 & 2009/08/19 & 6:06 & $11: 45$ & 5.7 \\
\hline $2009 / 08 / 20$ & $4: 29$ & $8: 50$ & 4.3 & $2009 / 09 / 12$ & 4:00 & $12: 46$ & 8.7 & $2009 / 09 / 13$ & $4: 36$ & $5: 46$ & 1.3 \\
\hline $2009 / 09 / 16$ & 4:00 & $5: 46$ & 1.7 & $2009 / 09 / 21$ & $4: 40$ & $6: 30$ & 1.8 & $2009 / 09 / 23$ & $3: 43$ & $13: 00$ & 9.2 \\
\hline $2009 / 09 / 24$ & $3: 43$ & $13: 00$ & 9.1 & $2009 / 09 / 25$ & $3: 25$ & $10: 18$ & 6.8 & $2009 / 09 / 26$ & $3: 50$ & $8: 02$ & 4.2 \\
\hline $2009 / 10 / 06$ & $3: 36$ & $5: 25$ & 1.8 & $2009 / 10 / 07$ & $7: 07$ & $12: 55$ & 5.8 & 2009/10/09 & $4: 15$ & $5: 33$ & 1.3 \\
\hline $2010 / 02 / 22$ & 4:04 & $5: 35$ & 1.6 & $2010 / 03 / 06$ & $4: 35$ & $13: 50$ & 8.3 & $2010 / 03 / 07$ & $3: 13$ & $6: 10$ & 3.0 \\
\hline $2010 / 03 / 18$ & $4: 19$ & $12: 10$ & 7.8 & $2010 / 03 / 20$ & $4: 52$ & $6: 44$ & 1.9 & $2010 / 05 / 07$ & $10: 42$ & $12: 32$ & 1.8 \\
\hline 2010/05/09 & $5: 23$ & $11: 34$ & 6.2 & 2010/05/11 & $5: 03$ & $11: 30$ & 6.5 & $2010 / 05 / 12$ & $6: 30$ & $9: 28$ & 3.0 \\
\hline $2010 / 05 / 14$ & $5: 03$ & $9: 52$ & 4.8 & $2010 / 05 / 22$ & $5: 26$ & $7: 21$ & 2.0 & $2010 / 06 / 06$ & $5: 50$ & $6: 00$ & 0.2 \\
\hline $2010 / 06 / 14$ & $6: 45$ & 7:04 & 0.3 & 2010/07/07 & $6: 16$ & $10: 52$ & 4.6 & $2010 / 07 / 08$ & $5: 43$ & 11:04 & 5.3 \\
\hline 2010/07/09 & $7: 05$ & 11:01 & 4.0 & $2010 / 07 / 14$ & 8:06 & 11:07 & 3.0 & $2010 / 07 / 15$ & $5: 42$ & $11: 18$ & 5.6 \\
\hline $2010 / 07 / 16$ & $5: 29$ & $10: 17$ & 4.8 & $2010 / 07 / 17$ & $6: 28$ & $11: 13$ & 5.8 & $2010 / 07 / 18$ & $5: 21$ & $11: 12$ & 5.9 \\
\hline $2010 / 07 / 19$ & $5: 14$ & $10: 15$ & 5.0 & $2010 / 07 / 20$ & $5: 48$ & $9: 12$ & 3.5 & $2010 / 07 / 21$ & $5: 25$ & $9: 36$ & 4.2 \\
\hline $2010 / 07 / 24$ & $5: 11$ & $11: 33$ & 6.3 & $2010 / 07 / 25$ & $5: 01$ & $11: 33$ & 6.5 & $2010 / 07 / 26$ & $4: 56$ & $11: 37$ & 6.6 \\
\hline $2010 / 07 / 27$ & $5: 01$ & $11: 28$ & 6.5 & $2010 / 07 / 28$ & $5: 59$ & $11: 00$ & 5.0 & $2010 / 07 / 29$ & $5: 17$ & $9: 48$ & 4.5 \\
\hline $2010 / 07 / 30$ & $5: 24$ & 11:11 & 5.8 & $2010 / 07 / 31$ & $5: 03$ & $11: 30$ & 6.5 & 2010/08/12 & $5: 26$ & $9: 17$ & 4.0 \\
\hline 2010/08/13 & $4: 45$ & $9: 35$ & 4.9 & 2010/08/14 & $5: 03$ & $11: 30$ & 6.5 & $2010 / 08 / 15$ & $4: 26$ & $12: 06$ & 7.5 \\
\hline $2010 / 08 / 16$ & $4: 57$ & 12:03 & 4.9 & $2010 / 08 / 25$ & $4: 45$ & $12: 23$ & 7.6 & $2010 / 08 / 26$ & $4: 50$ & 12:01 & 7.1 \\
\hline 2010/10/01 & $3: 36$ & $9: 23$ & 5.8 & $2010 / 10 / 03$ & $4: 56$ & $5: 21$ & 0.4 & 2010/10/07 & $2: 30$ & $13: 40$ & 11.2 \\
\hline $2010 / 10 / 14$ & $2: 27$ & $9: 28$ & 7.0 & 2010/10/17 & $2: 50$ & $7: 50$ & 5.0 & $2010 / 10 / 20$ & $3: 21$ & $13: 44$ & 10.3 \\
\hline $2010 / 10 / 21$ & $2: 50$ & $13: 52$ & 11.0 & 2010/11/04 & $1: 41$ & $13: 21$ & 11.7 & $2010 / 11 / 22$ & $5: 17$ & 9:04 & 3.8 \\
\hline $2010 / 11 / 24$ & $0: 50$ & $7: 19$ & 6.5 & $2010 / 12 / 04$ & 8:02 & 13:07 & 5.0 & $2010 / 12 / 05$ & 1:06 & $6: 59$ & 6.0 \\
\hline
\end{tabular}

Notes. All times are UTC.

the sodium region, one should keep in mind that because we have not corrected for geometric dilution, our reported "density" will overrepresent the density near the bottom of the sodium region, and underrepresent the density near the top, by as much as $20 \%$.

From a visual analysis of all nights with sodium layer data (see also the additional sodium maps in Fig. 1 of Pfrommer et al. 2009), the only clear conclusion that can be drawn about the general behavior of the sodium density structure is that it is highly variable. Each night reveals different structures on all time scales. Therefore, caution is needed when using any kind of "typical" data set to test the behavior of an AO-system in a laboratory environment or in a simulation. Nevertheless, an attempt was made to divide the vast amount of data into distinct types of profile shapes.

Seven distinct classes of profiles were identified, based on the shape of the density profiles. These are illustrated in Fig. 2. 
A\&A 565, A102 (2014)

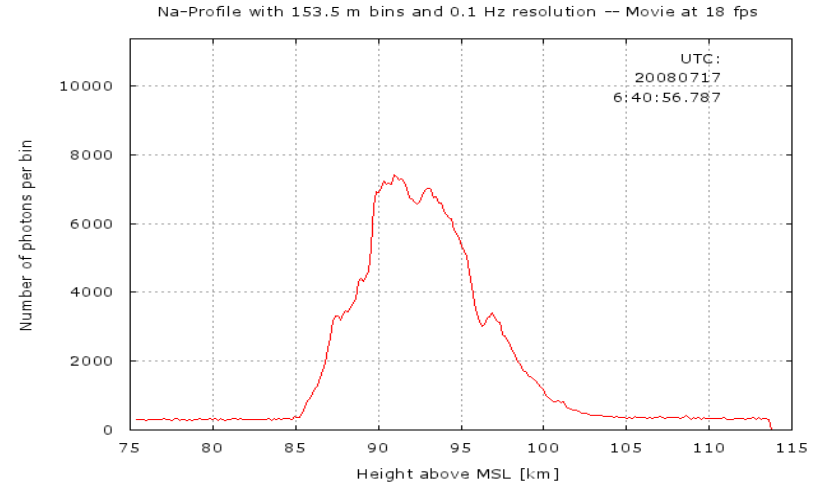

(a) Single peak

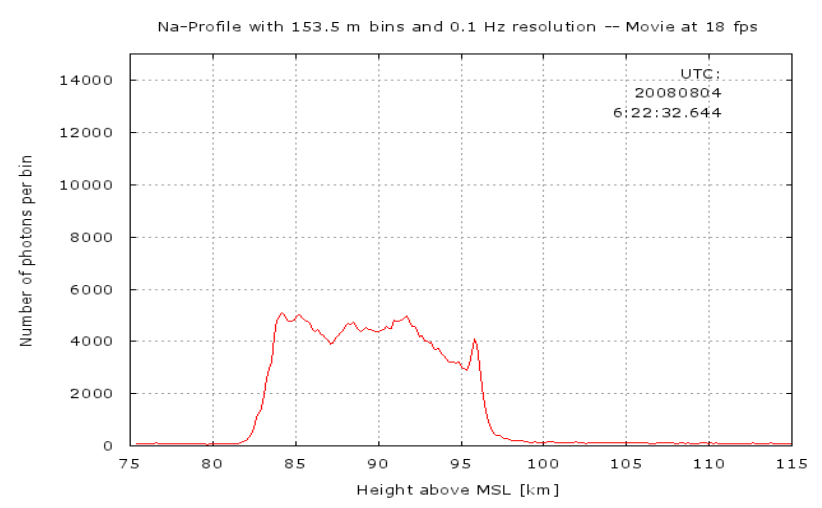

(c) Top hat

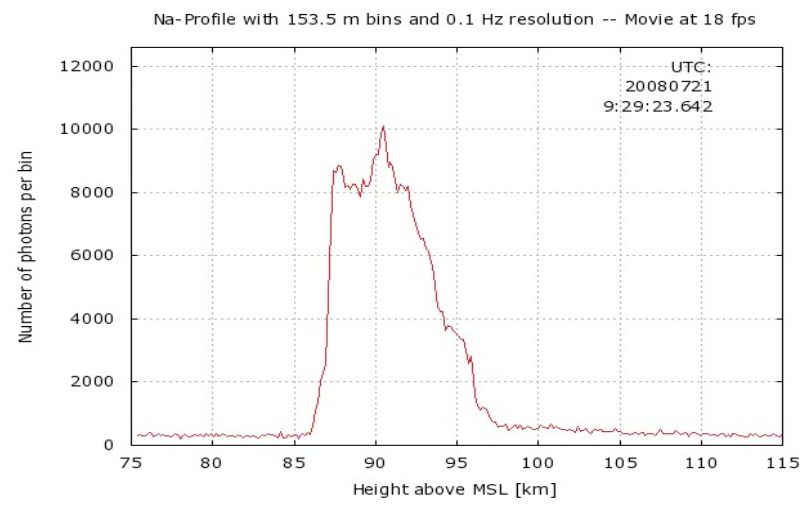

(e) Very narrow

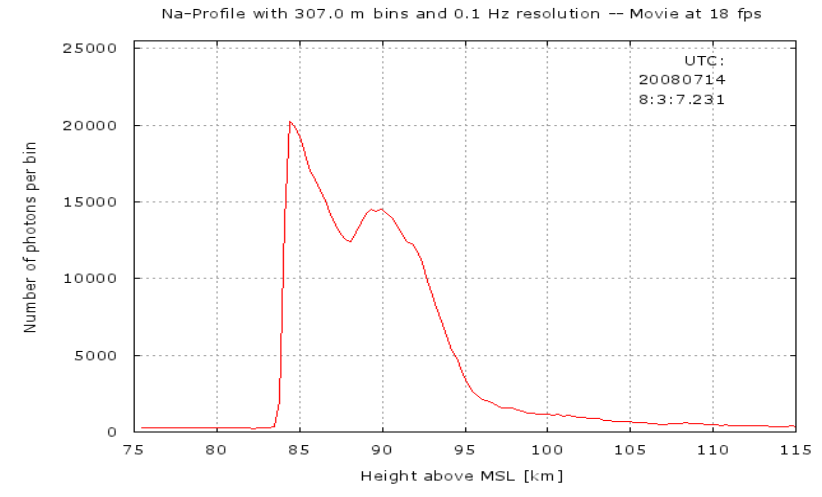

(b) Double peak

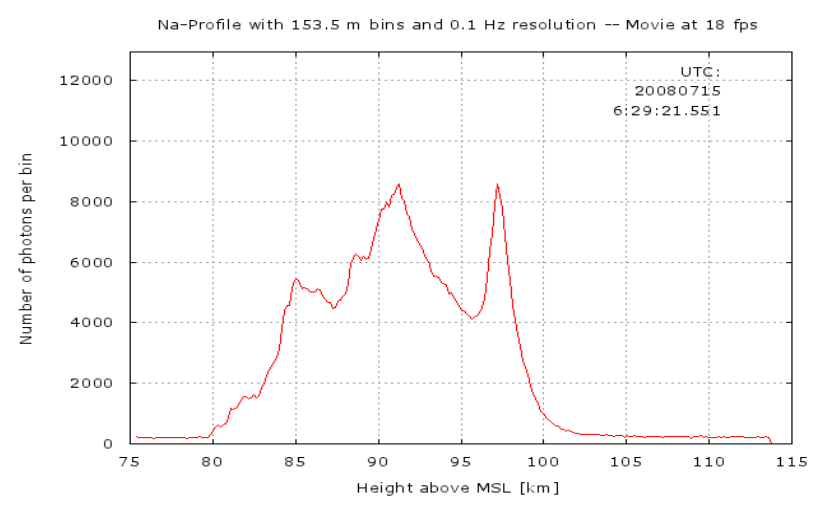

(d) Very wide

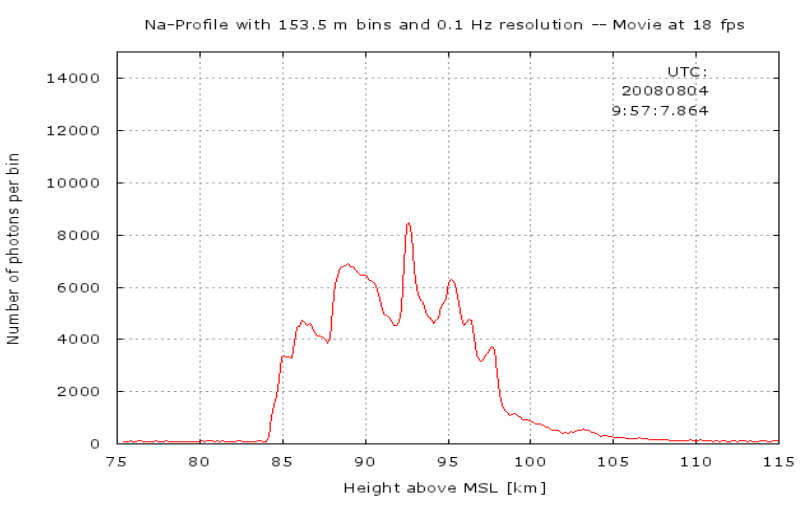

(f) Multi peak

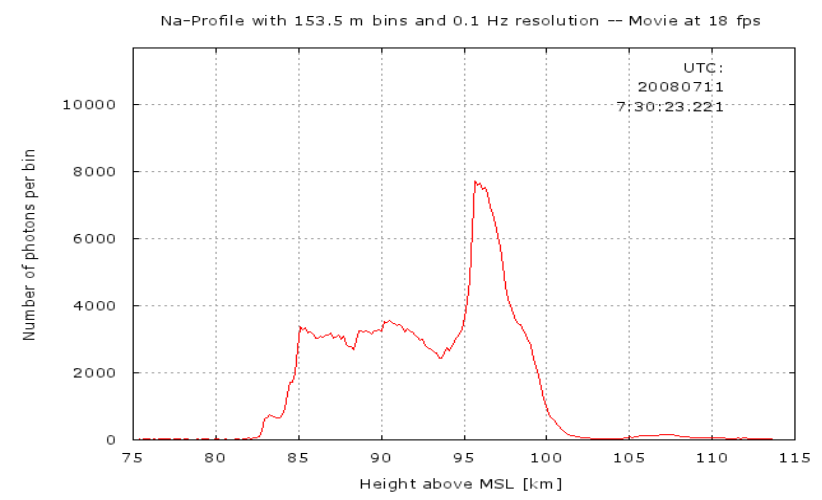

(g) Top hat with peak

Fig. 2. Classification of sodium density profile shapes. 
T. Pfrommer and P. Hickson: High resolution mesospheric sodium properties for adaptive optics applications

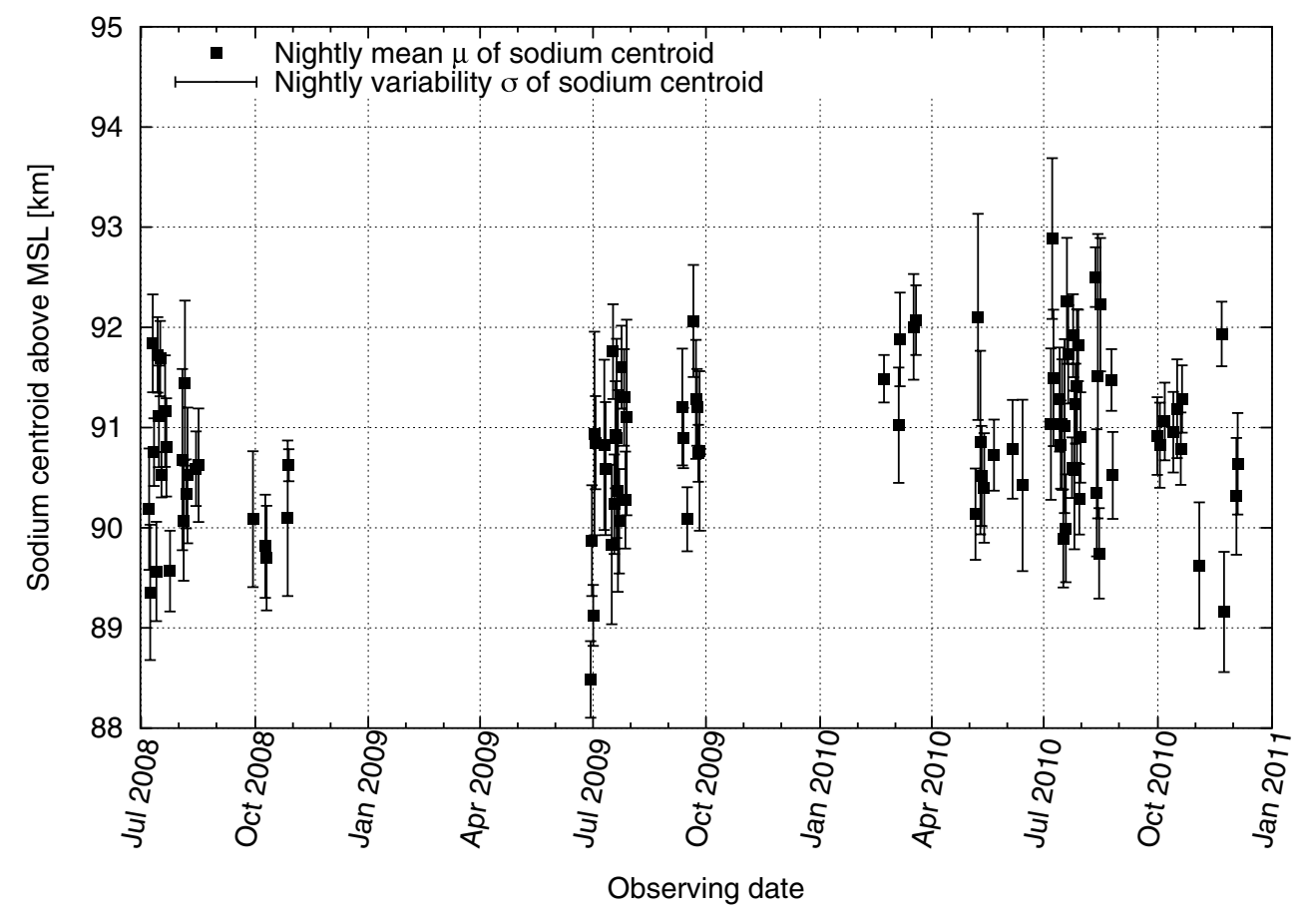

Fig. 3. Time evolution of the sodium centroid altitude. The points indicate the mean value for a given night and the error bars show the standard deviation of all values measured during the night.
The frequency of occurrence for each of the different shapes is comparable. The figures show photon counts averaged in $10 \mathrm{~s}$ temporal bins and $153.5 \mathrm{~m}$ vertical bins. It is evident from these figures that smooth Gaussian-shaped profiles rarely occur. Rather, there is much substructure within the profiles, which typically changes on timescales of less than an hour.

\subsection{Mean altitude}

A robust quantity that does not depend on the variable uplink laser power is the flux-weighted mean altitude. This parameter, defined by

$a(t)=\frac{\int_{0}^{\infty} F(z) z \mathrm{~d} z}{\int_{0}^{\infty} F(z) \mathrm{d} z}$

is easily measured and does not require any knowledge of the absolute sensitivity or efficiency of the lidar system. The nightly means are plotted as time series of observing dates in Fig. 3. The variations within a given night, indicated by the error bars, are usually quite large compared to the changes from one night to another. They subtend a range from about $1 \mathrm{~km}$ to $2 \mathrm{~km}$ with outliers up to $3 \mathrm{~km}$ and down to $500 \mathrm{~m}$. Its distribution is over one night mostly Gaussian in shape, but sometimes weather conditions in the mesosphere cause drastic changes. This situation is illustrated in Fig. 4, where the distribution functions of mean altitude are shown for two different nights in 2008. It can be seen that on July 18th, the distribution is very nearly Gaussian, while on August 6th it has two distinct peaks, indicating more complex dynamics on that night.

The flux-weighted mean sodium altitude, averaged over all our data, is $90.8 \pm 0.1 \mathrm{~km}$, and the distribution for all data is shown in Fig. 5. Note that this value (as for any mean altitude stated in this paper) is not corrected for geometric dilution and thus represents the mean altitude as seen by an AO system.

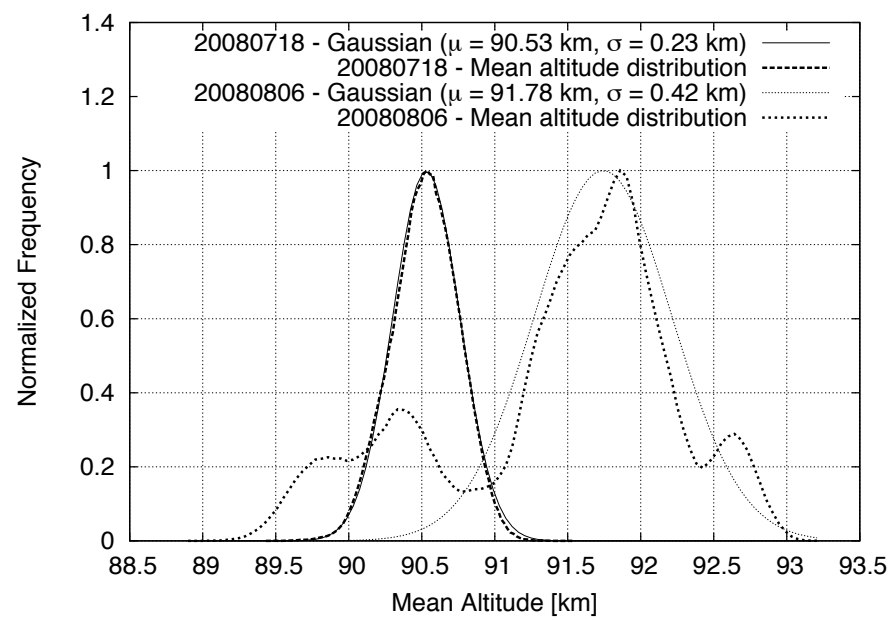

Fig. 4. Distribution of the sodium centroid altitude for two nights, illustrating the variability that can occur. The best-fitting Gaussian curves are also shown.

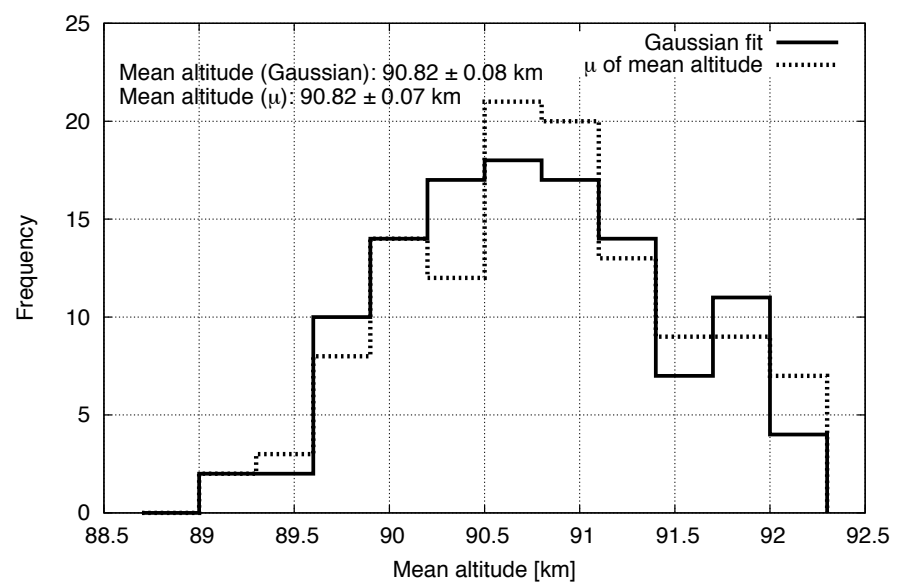

Fig. 5. Distribution of the sodium centroid altitude for all data. 


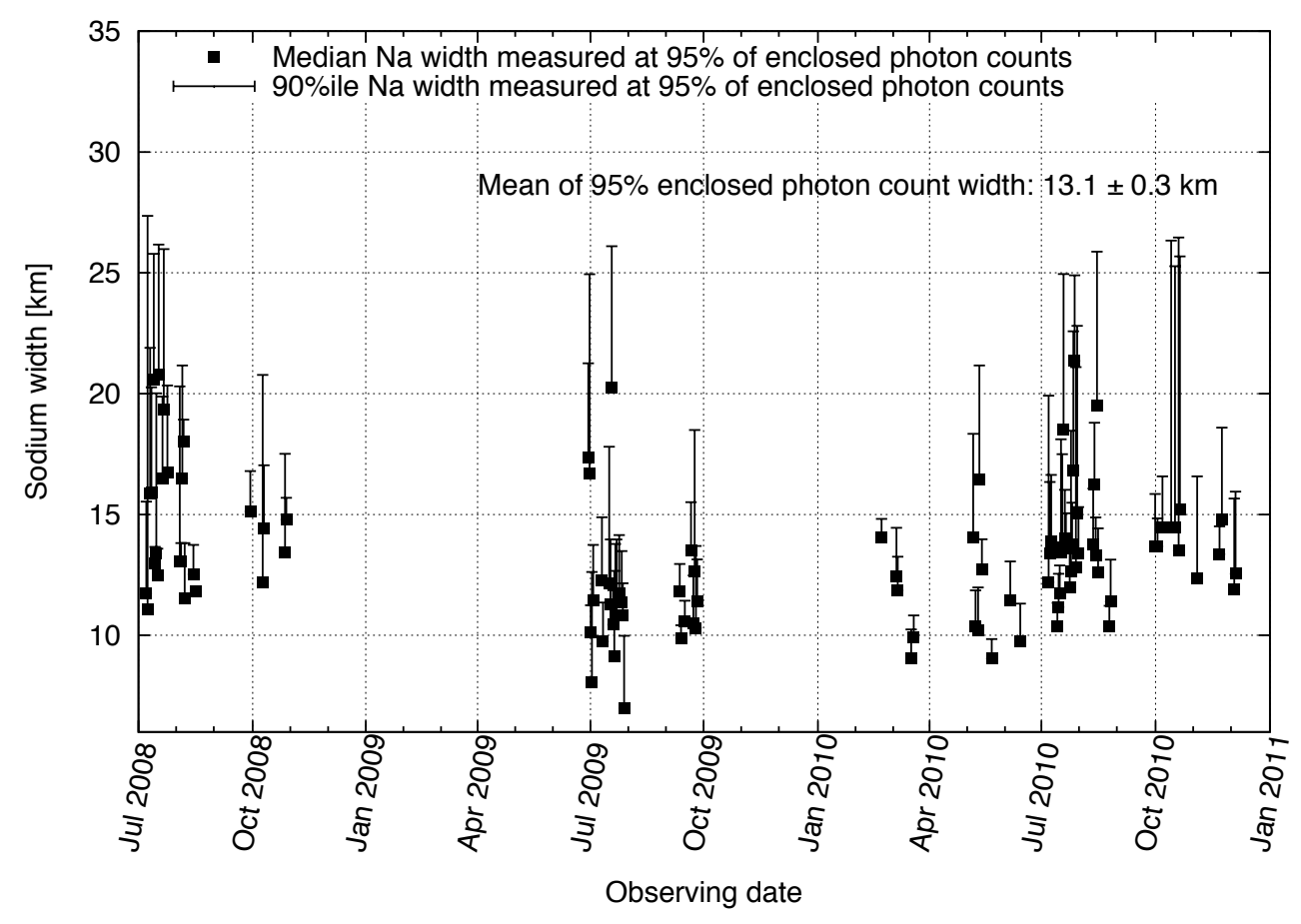

Fig. 6. Time evolution of the sodium width. The sodium width based on medium altitude range containing $95 \%$ of the returned photons is plotted vs. date of observation. The error bars show the 90th percentile range of this parameter.

\subsection{Sodium width}

A number of methods for defining the sodium width have been proposed in the literature. A simple measure is the square root of the second moment of the sodium density profile about the mean, $\sigma_{z}$. For a Gaussian profile, this quantity is related to the FWHM $w$ by $w=2 \sqrt{2 \ln (2)} \sigma_{z} \simeq 2.355 \sigma_{z}$.

Even though the profile is rarely Gaussian, this parameter nevertheless provides a good indication of the nightly variations.

Alternatively, one can use the altitude interval containing a specified percentile of enclosed photon counts. This gives an indication of the signal that is lost in an AO system if the altitude range of the WFS is limited, and can be used to assess the potential bias in the resulting mean altitude. A third parameter is the altitude range within which the return flux is greater than a specified percentage of the peak flux. We refer to these three measures as "second moment", "enclosed energy", and "flux threshold" respectively.

In the following, the enclosed energy parameter is used for comparison of different nights and to give absolute sodium width information. In Fig. 6, the median 95\% width of enclosed flux for each observing night is plotted. Also shown is the 90th percentile variation of this parameter, indicated by the one-sided error bar. Changes in the sodium width within each night generally exceed night-to-night and seasonal variations. This is consistent with the variations seen in the centroid altitude (Fig. 3).

The distributions for all nights for three different percentages is shown in Fig. 7. The mean sodium width, for the 3-year data set, is found to be $11.3 \pm 0.2 \mathrm{~km}$ for $90 \%$ enclosed energy, $13.1 \pm 0.3 \mathrm{~km}$ for $95 \%$ and $15.8 \pm 0.4 \mathrm{~km}$ for $99 \%$ enclosed energy.

In all these distributions it is clear that the sodium width is changing significantly from one night to another and the entire range of sodium layer thicknesses varies by as much as 12 to $14 \mathrm{~km}$. Not only is the width changing on a nightly basis, the variation within one night is also strong. This can also be seen in Fig. 6.

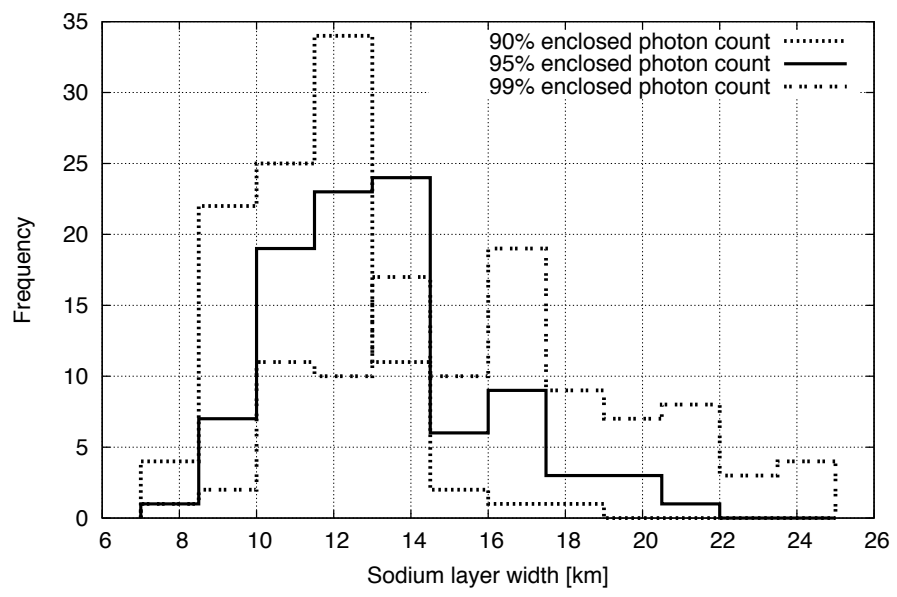

Fig. 7. Distribution function of sodium width. The curves show the distribution of the nightly median interval of altitude that contains $90 \%$, $95 \%$ and $99 \%$ of the returned photons.

The altitudes of the upper and lower ends of the sodium region were estimated by determining the altitude at which the sodium flux rises more than 5 standard deviations above the average background level. The results are shown in Fig. 8. Averaged over all data, the mean lower edge altitude is $81.67 \pm 0.12 \mathrm{~km}$, and the mean upper edge altitude is $104.86 \pm 0.30 \mathrm{~km}$.

It is instructive to bin the sodium profiles to a size that matches the angular sampling of a typical AO WFS. For example, for the E-ELT, a WFS pixel size of $0.5^{\prime \prime}$ corresponds to approximately $0.5 \mathrm{~km}$ vertical height for subapertures that are farthest from the laser launch telescope. The enclosed energy parameter gives similar results on average, but the $90 \%$ of peak flux parameter now gives a mean sodium width of almost $20 \mathrm{~km}$. Thus, the latter method appears to be more sensitive to binning effects.

This large variation in sodium widths provides challenges for AO design which must balance the greater detector read noise, that increases with the number of pixels, against sampling errors. 
T. Pfrommer and P. Hickson: High resolution mesospheric sodium properties for adaptive optics applications

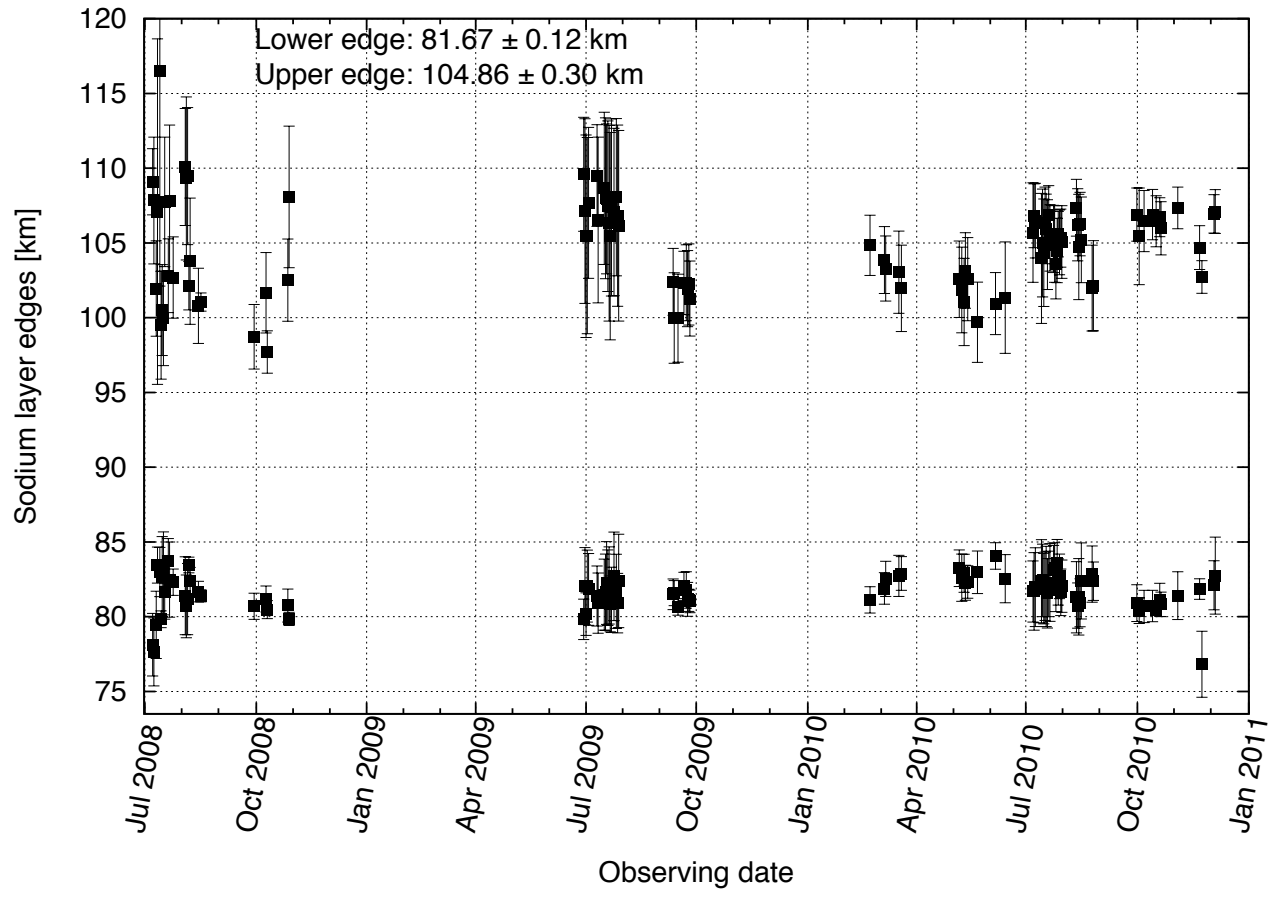

Fig. 8. Time evolution of the lower and upper edge of the sodium region.

\subsection{Mean-altitude power spectrum}

To investigate the dynamics of the sodium layer, a fundamental statistic is the temporal power spectral density of the mean altitude. When combined with the response function of an AO system, this spectrum determines the direct impact of altitude variations on system performance. A typical (one-sided) sodium density temporal power spectral density (PSD) was shown in Pfrommer \& Hickson (2010) (Fig. 2). Similar spectra were computed for each night of the present data set. These are all well represented by a power law

$P_{a}(v)=\alpha v^{\beta}$,

where $v$ is the fluctuation frequency, but with variations in the parameters $\alpha$ and $\beta$. For each night, these two parameters were estimated by means of a Markov-chain Monte Carlo (MCMC) fitting routine. In this process, the background photon noise present in the spectrum was estimated from the actual photon counts, by applying Poisson statistics. However this was generally not sufficient to account for all of the white noise present in the spectrum. Therefore, in the fitting process three parameters are varied. These are $\alpha$ and $\beta$, and the background noise amplitude. Only one power spectrum was calculated for each night. Results are given in the following paragraphs.

The normalization amplitude $\alpha$ of the temporal meanaltitude power spectrum is an important parameter for $\mathrm{AO}$ as it most closely represents the power with which the mean altitude varies at the frequencies relevant for AO. The distribution of this parameter is shown in Fig. 9. For the entire data set, the mean amplitude is $34.4_{-4.8}^{+5.6} \mathrm{~m}^{2} \mathrm{~Hz}^{-1}$. The yearly averages are 28.5 $5_{-6.0}^{+7.6} \mathrm{~m}^{2} \mathrm{~Hz}^{-1}$ for $2008,19.4_{-3.9}^{+4.8} \mathrm{~m}^{2} \mathrm{~Hz}^{-1}$ for 2009 , and $51.5_{-11.0}^{+13.9} \mathrm{~m}^{2} \mathrm{~Hz}^{-1}$ for 2010 .

The time series of $\alpha$ is shown by the lower series of points, and right-hand scale of Fig. 11. The error bars indicate the standard deviation of the marginalized posterior-probability function, from the MCMC analysis. This plot shows that the amplitude can change over a period of days by more than an order of magnitude. Generally, the change is gradual, exhibiting a continuous trend, which suggests that the variation is due to slowly

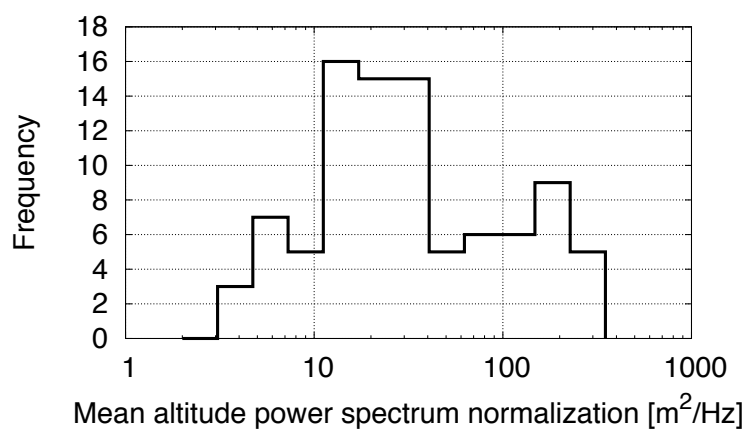

Fig. 9. Distribution of the mean-altitude power spectrum normalization amplitude $\alpha$.

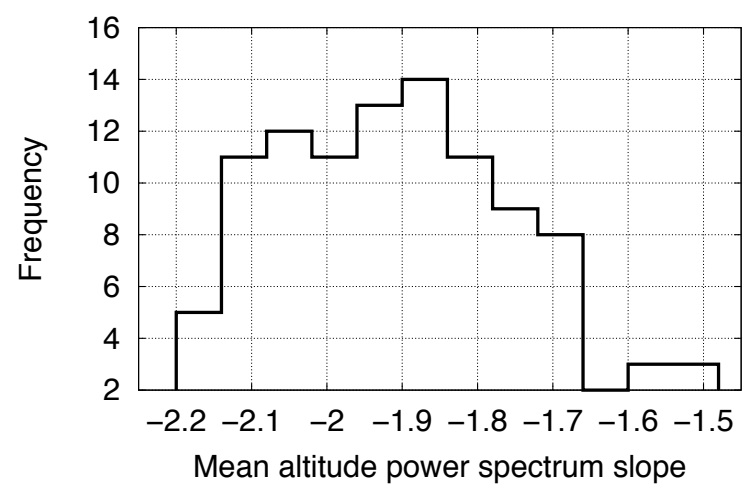

Fig. 10. Distribution of the mean-altitude spectral index $\beta$.

changing physical parameters on time scales of at least days. However, sudden variations are sometimes seen that break this pattern.

The distribution of the spectral index $\beta$ is shown in Fig. 10, and by the upper points and left-hand scale in Fig. 11. For the whole data set, the mean slope is $-1.87 \pm 0.02$. The yearly means 


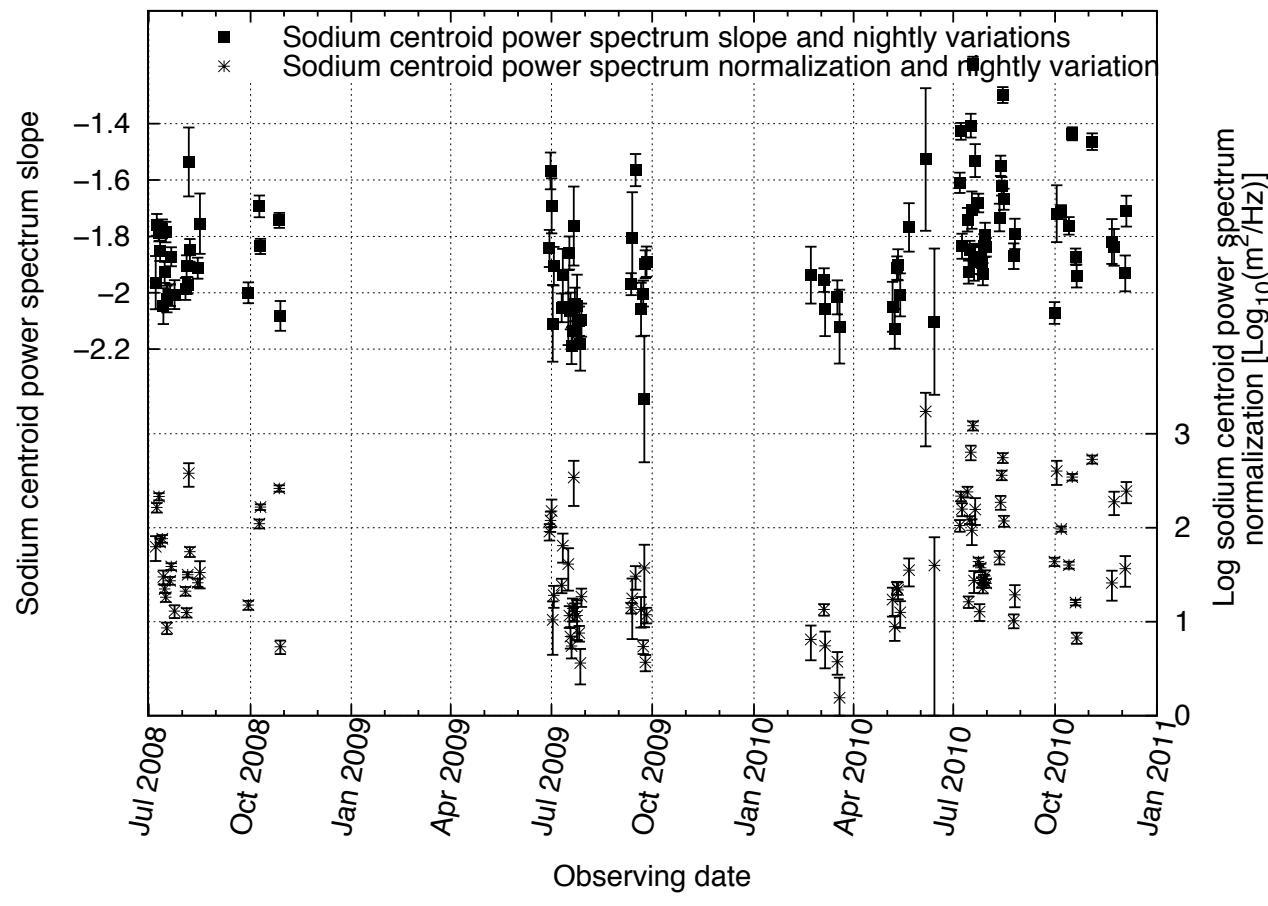

Fig. 11. Time evolution of the amplitude and spectral index of the temporal PSD of sodium centroid-altitude fluctuations. are $-1.93 \pm 0.03$ for $2008,-1.97 \pm 0.04$ for 2009 , and $-1.78 \pm$ 0.03 for 2010 . As with the amplitude, consecutive nights usually show similar slopes, but sudden changes can occur.

It is interesting to look for correlations between the amplitude and slope to see if there is a general trend, and hence a pivot point or small frequency range around which the variation in power is smallest. This is found at frequencies between $10^{-3}$ and $10^{-2} \mathrm{~Hz}$. In this frequency range, the spread in power is approximately 1.5 orders of magnitude. At the high frequency end above $10 \mathrm{~Hz}$, the power varies by more than 4 orders of magnitude.

\subsection{Centroid velocities}

Another quantity of interest is the centroid velocity $v_{a}(t)=\dot{a}(t)$. By the Fourier derivative theorem, its Fourier transform $\tilde{v}_{a}(v)$ is $2 \pi \mathrm{i} v \tilde{a}(v)$, hence the centroid velocity power spectrum is related to the centroid altitude power spectrum by

$$
P_{v}(v)=4 \pi^{2} v^{2} P_{a}(v)
$$

From this one can calculate the mean-square centroid velocity over any temporal bandwidth by multiplying by the appropriate filter function and integrating over frequency. For example, the mean square centroid velocity averaged over time $T$ is given by

$$
\begin{aligned}
\sigma_{v}^{2}(T) & =\frac{1}{\pi^{2} T^{2}} \int_{0}^{\infty} P_{v}(v)[\sin (\pi v T)]^{2} v^{-2} \mathrm{~d} v \\
& =\frac{2 \alpha \Gamma(\beta+1) \sin (\pi \beta / 2)}{(2 \pi)^{\beta+1}} T^{-\beta-3}, \quad(-3<\beta<-1) .
\end{aligned}
$$

This approach is more robust than direct centroid velocity measurements, which are subject to errors from differentiation of noisy mean altitude profiles. With the mean parameters $\alpha$ and $\beta$ from the whole data set, and a time interval $T$ of $1 \mathrm{~s}$, the RMS centroid velocity is about $24 \mathrm{~ms}^{-1}$. Over a time interval of $10 \mathrm{~s}$, the RMS centroid velocity is reduced to about $8 \mathrm{~ms}^{-1}$.

\subsection{Transient events}

It has been known for many years that meteor ablation trails can cause sudden spikes in sodium lidar returns as the trail is carried across the laser beam by the mesospheric wind (Kane \& Gardner 1993; von Zahn et al. 1999). These events frequently appear in our data, typically near the lower boundary of the sodium region. Their durations range from sub-second to several tens of seconds. The vertical extent of the affected region is less than a few hundred meters in height. In previous publications (Pfrommer et al. 2009; Pfrommer \& Hickson 2010), we reported subsecond meteor trails and discussed their impact on the mean altitude time series. An example of such an event is shown in Fig. 12, where the meteor trail and its effect on the mean-altitude time series is easily seen. We see events that caused the mean altitude to change by as much as $1 \mathrm{~km}$ over less than $1 \mathrm{~s}$ time span.

In order to study these events in detail and determine their statistical properties, a computer program was developed to automatically detect these events and measure their properties. To detect the events, the program applies a matched filter to the raw unbinned two-dimensional flux (photon count) maps. This is done by convolving the map with a high-pass filter composed of the difference between two two-dimensional Gaussian functions. A wide Gaussian is subtracted from a narrow Gaussian to produce a filter that has zero mean. The narrow Gaussian matches the typical width of meteor trail profiles. The standard deviations of these two components were chosen to be $30 \mathrm{~m}$ and $400 \mathrm{~ms}$ respectively. This choice of parameters enhances features that are comparable to or smaller than the narrow component and rejects features larger than the wide component. In order to automatically detect meteor trails, a histogram of the flux in the filtered map is generated. If there are no meteors, a symmetric distribution around zero flux is expected. In the case of a meteor detection, a tail towards positive flux is present which allows for automatic detection. The region affected by the meteor can now be determined and the characteristics and parameters of the meteor trail are computed from the original unfiltered map. 
T. Pfrommer and P. Hickson: High resolution mesospheric sodium properties for adaptive optics applications
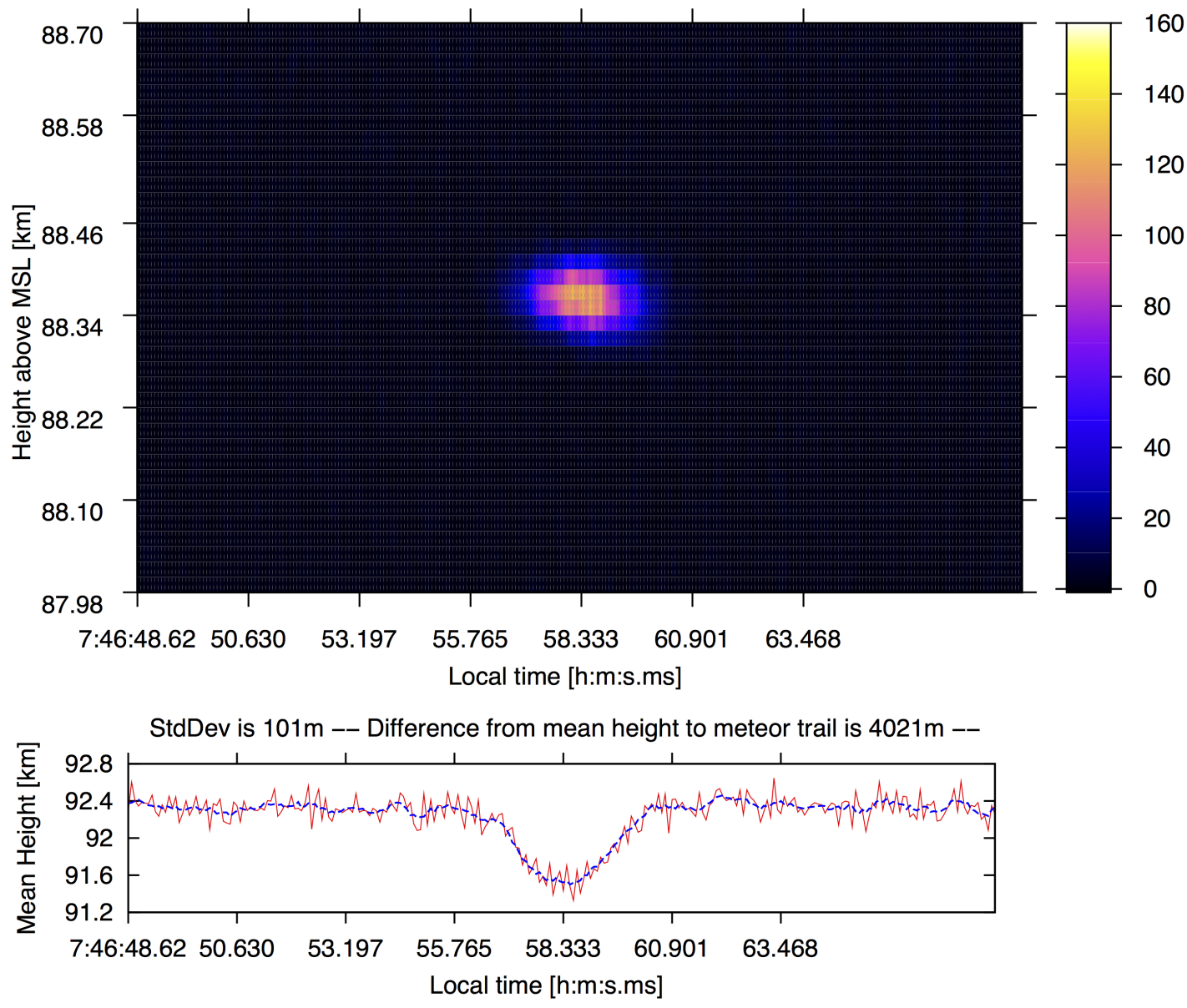

Fig. 12. Meteor ablation trail seen on the night of 12 th of September 2009

The most significant meteors, from the perspective of $\mathrm{AO}$, are those that change the mean altitude significantly in a short period of time. Specifically, we define a meteor to be "significant" if the mean altitude changes by more than $2 \sigma$, where $\sigma$ is the local standard deviation of the mean altitude within a time rage of $\pm 10 \mathrm{~s}$ from the event. Figure 13 shows the number of detected meteors per night (solid squares). The open squares show the number of significant meteors per night and stars indicate the respective fraction of significant meteors for each night. The fraction of significant meteors is usually around $20 \%$, but can be as high as $80 \%$. However some nights also show no detected significant events at all.

The rate distribution for the full data set is shown in Fig. 14.

It can be seen that the number of detected meteors is roughly uniformly distributed up to a maximum of about 15 meteors per night, with noticeably fewer above this level. A few nights showed more meteor events up to a maximum of about 40 . Roughly half of the nights had no significant meteor event, and a quarter had only one significant event. The other observing nights (about a quarter) had at least 2 significant meteor events ranging up to a maximum of 12 per night. On average, 2 meteors per hour are detected, but at some nights up to about 9 meteors per hour are seen. Occasionally up to 20 meteors per hour have been detected. The significant meteor events occur usually very sporadically and the distribution reveals an average of around 0.2 meteors per hour. About 20 nights showed more than one significant meteor event per hour with the occasional night having more than 5 significant events per hour.

The altitude distribution of the meteors peaks around $85 \mathrm{~km}$ (or $\sim 6 \mathrm{~km}$ from the local mean altitude), as shown in Figs. 15. The significant meteors follow the same distribution. Most of the detected meteor events last around $1 \mathrm{~s}$, but the tail of the time extent distribution reaches up to $14 \mathrm{~s}$. The significant meteor subsample shows a double peak behavior. One part is very confined, less than $400 \mathrm{~ms}$ in time extension, and the other peak is around $2 \mathrm{~s}$. As expected from definition of this subsample, the tail of the significant meteor distribution falls off less quickly, accounting for the fact that a larger fraction of the more extended meteors cause a significant dip in the mean altitude. The double peak behavior for significant meteors is also seen in the distribution of their height extensions and thus shows the influx of two distinctly different meteor samples. In the vertical dimension, the confined height extension range is detected to be less than $50 \mathrm{~m}$, and the group with larger extensions shows mean values around $150 \mathrm{~m}$. Meteors have been detected that extend up to $600 \mathrm{~m}$ in height. For the full meteor sample, the distribution peaks around 50 to $60 \mathrm{~m}$ with no second peak being present.

For the significant meteor subsample, the distribution of the variation in the sodium centroid altitude is shown in Fig. 16. This parameter is of interest for $\mathrm{AO}$ design together with the rate. As can be seen in Fig. 16, most of the significant meteors change the centroid by about $200 \mathrm{~m}$, but a significant number of meteors can change the centroid by as much as $1 \mathrm{~km}$.

\section{Discussion}

Variability in the sodium region is governed by a number of diverse physical processes. The upper limit of the sodium layer is defined by the recombination of sodium ions, produce by meteoric ablation, with free electrons from the ionosphere and the plasma generated during the air-braking process from entering meteors. The removal of sodium at the lower limit of 


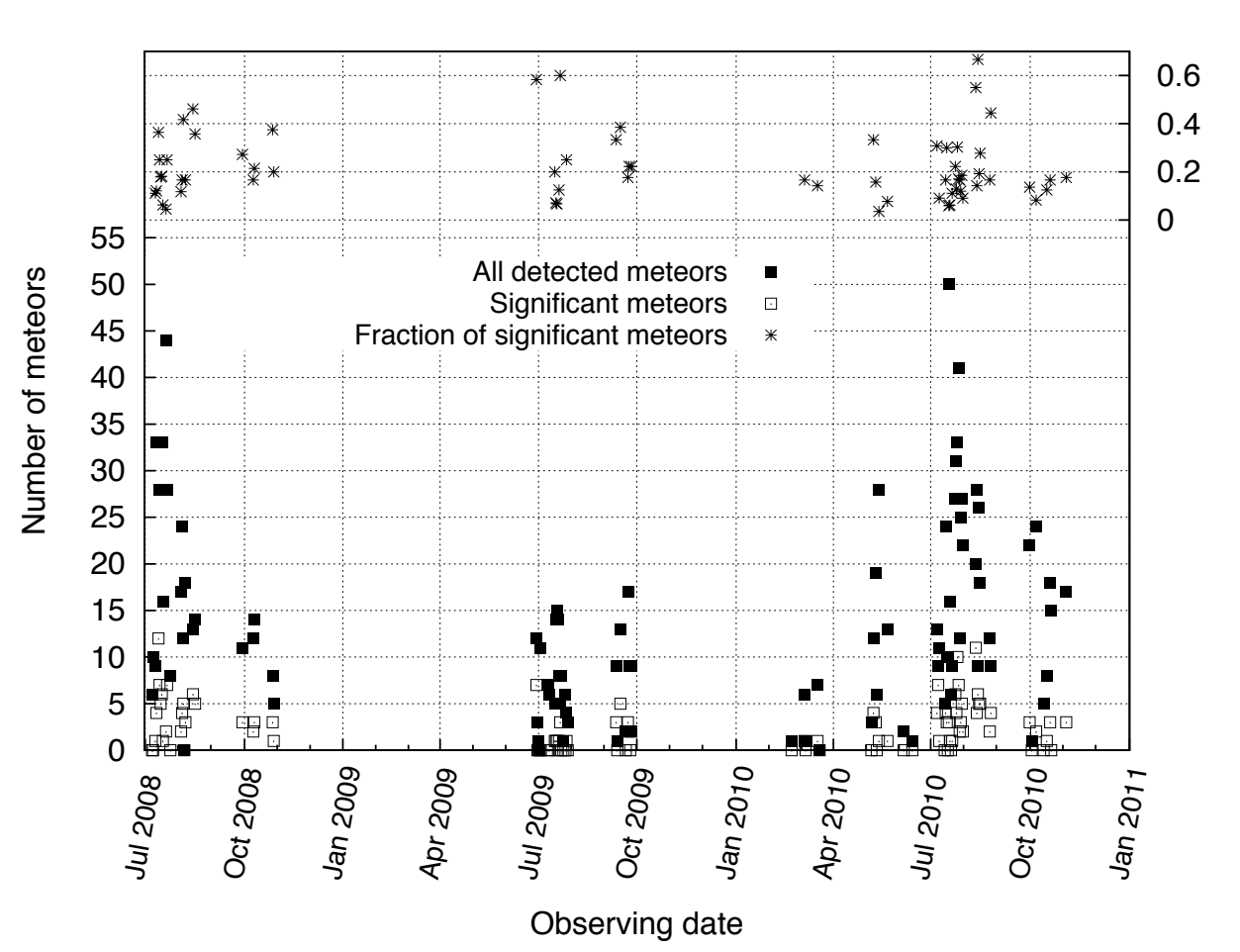

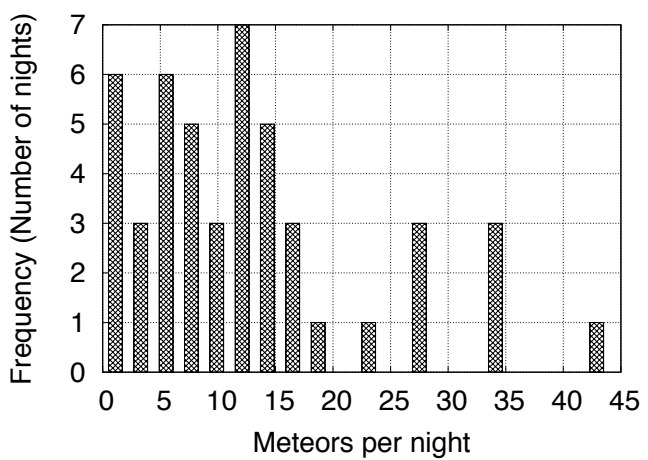

(a) Number of meteors per night

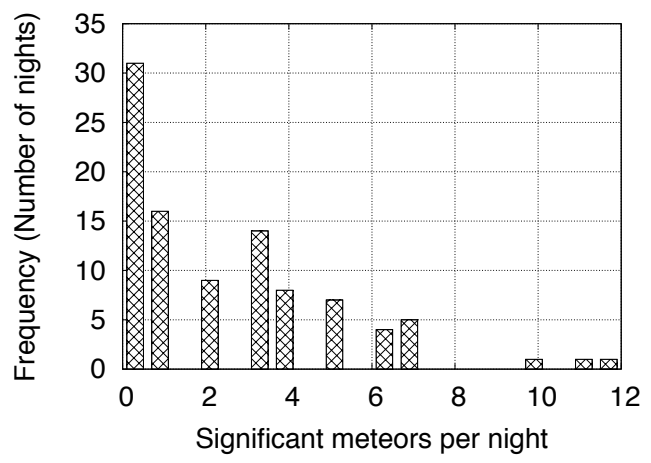

(b) Number of significant meteors per night
Fig. 13. Meteor events vs. date.

Fig. 14. Distribution of the number of meteors per night and the number of significant meteors per night.

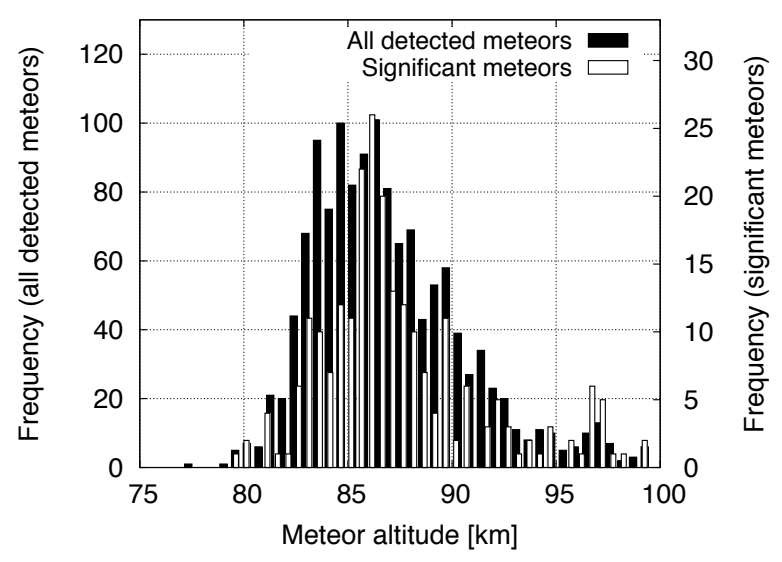

Fig. 15. Distribution of altitudes of observed meteor trails.

the sodium layer depends on free oxygen radicals and hence the ozone concentration. This reaction is temperature dependent and it seems above a threshold the removal mechanism is rapid and effective (Plane 2003). This results in usually much steeper sodium density profile shapes at the lower end of the sodium

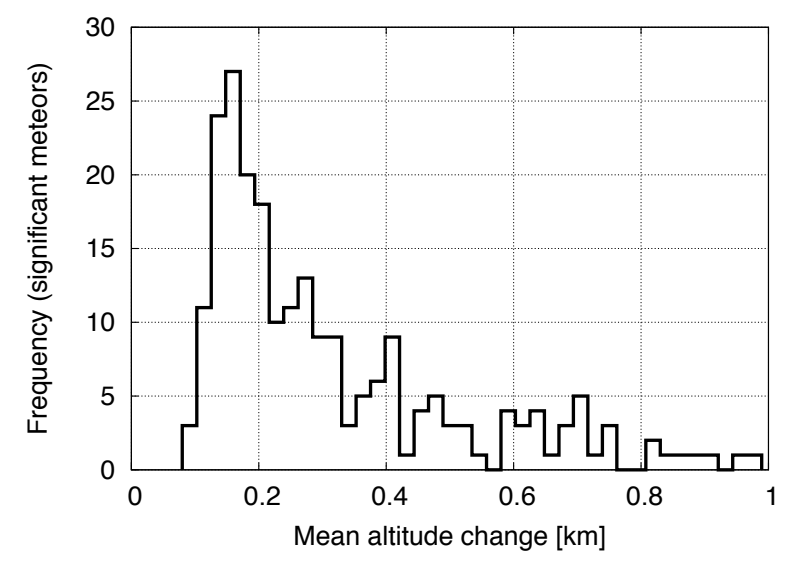

Fig. 16. Distribution of centroid altitude variations produced by significant meteor trails. Thus only those meteor events that change the centroid altitude by more than twice the local standard deviation of the centroid altitude time series are included in this distribution.

layer compared to the upper end. In addition to the meteor influx function (Ceplecha et al. 1998), the source function is also 
dependent on space weather which affects the electron density, and is therefore not constant (von Zahn 2005; Plane 2003). With the sodium atoms being about a factor of $10^{8}$ less abundant than air molecules, the sodium acts as tracer particle to highlight the underlying dynamic phenomena.

At $90 \mathrm{~km}$, the atmosphere is about a factor of $10^{6}$ less dense than at sea level. At such densities, gravity waves propagating upward from the troposphere reach amplitudes of several $\mathrm{km}$, become nonlinear and dissipate. The energy that they deliver is comparable to other driving factors such as tides, solar irradiance and wind. Wind speed and direction is determined primarily by global circulation. When combined with diurnal tidal flows, wind speeds can exceed $100 \mathrm{~ms}^{-1}$. The wind speed changes rapidly with altitude, with a shear as high as $60 \mathrm{~ms}^{-1}$ per $\mathrm{km}$ (Yuan et al. 2008).

If gravity waves dominate the structure in the region of interest, theory predicts distinct peaks in the height profile that appear as sodium clouds containing higher density in the structure maps (Liu \& Yi 2009). Such gravity waves are expected to exhibit a downward phase velocity, while their group velocity is directed upwards. Most detected sodium clouds show evidence of a general downward drift with time, also seen in Fig. 1, however other effects such as downward drift of atoms due to gravity may play a role, depending on the source rate. It is possible that some sodium clouds may originate from a single mg to g-sized meteoroid, whose neutralized ablation plume has been sheared by the wind, producing a stratified layer (Liu \& Yi 2009).

In the sodium layer multiple clouds exist whose structure evolves with time. It sometimes appears that the entire layer is built up out of these clouds rather than one or two clouds being superimposed on a smooth sodium background. The lifetime of an individual cloud is typically a few hours. Some clouds appear rapidly and can reach densities that are a factor of several times greater than the mean sodium background. These sporadic sodium layers can cause shifts in the mean sodium altitude of several $\mathrm{km}$ in just a few minutes.

Gravity waves also create oscillations in the sodium layers with periods of several minutes. These have the appearance of ripples in our maps, and can be seen in Fig. 1. These might originate from weather events such as storms in the tropopause that create waves on small spatial scales. These gavity waves appear as spatial and temporal oscillations in the sodium region. Their effect on the lidar maps is two fold. There is not only the direct temporal variation, but also that due to advection by the wind of spatial variations. These oscillations are seen to extend sometimes coherently throughout the sodium region.

On even smaller time scales, less than a few minutes, we often see turbulent structures such as Kelvin Helmholtz instabilities (Pfrommer et al. 2009) originating from the stratified shear flow. An example is shown in Fig. 17. Such instabilities are expected whenever the Richardson number is below 0.25 , as is often the case in the mesosphere.

The sodium layer width is important in determining the required field of view for subapertures in an AO WFS. While it is not essential that a wavefront sensor samples the entire elongated LGS, truncation or vignetting of the image introduces bias and uncertainty in the centroid altitude. Since the angular elongation of the LGS is a function of position in the pupil, truncation results in coupling of these errors to higher-order AO modes. Ideally, the WFS should be designed to accommodate the greatest possible LGS elongation, taking into account the seasonal variations. Also, fine structure in the sodium can affect the choice of WFS pixel size.

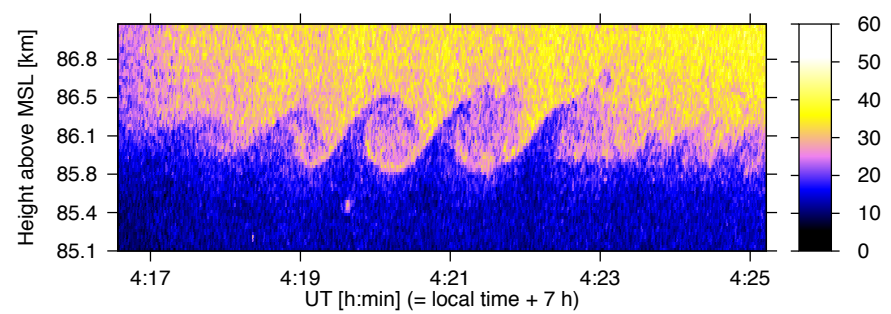

Fig. 17. Onset of Kelvin-Helmholtz instabilities in the sodium layer from the night of Sept. 12th 2009. Color-coded are again photo counts per bin.

The frequency and impact of meteors is another factor. AO control systems must be trained to identify and reject these transient events. In addition to the statistical analysis presented here, an attempt was made to correlate our data with meteor radar surveys in order to work towards predictive AO and observing scheduling. These surveys are sensitive primarily to $\mathrm{mg}$ $(100 \mu \mathrm{g})$ sized meteors (Brown et al. 2008, 2010). Different selection processes complicate such a comparison. The lidar detects ablation trails that are advected by wind. Hence our detection frequency depends on the trail dissipation rate and on the windspeed at the altitude of the trail. In contrast, radar gives a snapshot of the number of meteor trails per square kilometer over a certain time span. From the height and time distributions of the lidar meteor events, one could in principle estimate the wind speed by assuming that the ablation trail has a circular cross section. Even so the dissipation time is uncertain, so a direct comparison between the two data sets would still remain questionable. Thus, from this 3-year data set it is not yet possible to derive predictions regarding detected meteor frequency and known major meteor showers.

The most significant impact on $\mathrm{AO}$ comes from variations in the sodium centroid altitude. These translate directly to focus errors for any sodium AO system employing CW lasers. For a telescope of diameter $D$ at altitude $a_{0}$ observing at zenith angle $\zeta$, the piston-removed rms wavefront error (averaged over the telescope aperture) resulting from an altitude variation $\Delta a$ is (Herriot et al. 2006; Davis et al. 2006)

$\sigma_{\mathrm{wfe}}=\frac{D^{2} \cos \zeta}{16 \sqrt{3}\left(a-a_{0}\right)^{2}} \Delta a$.

For E-ELT observing near the zenith, this corresponds to a wavefront error of $\sim 8 \mathrm{~nm}$ per meter of altitude variation. At low frequencies, NGS can be used to determine the atmospheric focus correction, but at high frequencies photon noise degrades that signal so the focus correction must be determined from sodium LGS. The error in this signal depends on the integral of the mean altitude power spectrum above the guide star transition frequency. This integral is determined by the amplitude and index of the power spectrum.

The impact of sodium variability will depend to some degree on the details of the AO implementation. AO modes, including focus, are estimated by a fit using data from all WFS subapertures. For a given signal-to-noise ratio $(\mathrm{S} / \mathrm{N})$ per subaperture, the error in estimating any mode will decrease in proportion to the square root of the number of subapertures. This applies to focus estimation using NGS. If the telescope aperture is increased, the temporal frequency with which the focus is estimated from NGS, $v_{\text {NGS }}$, can be increased. For a given $\mathrm{S} / \mathrm{N}$, this frequency will be proportional to the aperture area, $v_{\mathrm{NGS}} \propto A \propto D^{2}$, if 
photon statistics is the dominant source of noise. At higher frequencies, the focus estimate must be derived primarily from the LGS. The variance in this estimate is given by

$\sigma_{\mathrm{LGS}}^{2} \propto D^{4} \cos ^{2} \zeta \int_{0}^{\infty} P_{a}(v)\left|H_{\mathrm{rej}}(v)\right|^{2} \mathrm{~d} v$,

where $H_{\text {rej }}(v)$ is the rejection transfer function of the AO system and the square of the altitude variation $\Delta a$ from Eq. (6) is replaced by the integral over the mean altitude power spectrum. Our results show that $P_{a}(v) \propto v^{\beta}$, so the variance can be written in the form

$\sigma_{\mathrm{LGS}}^{2} \propto D^{4} \cos ^{2} \zeta \nu_{\mathrm{NGS}}^{\beta+1} \int_{0}^{\infty} x^{\beta}\left|H_{\mathrm{rej}}(x)\right|^{2} \mathrm{~d} x$,

where $x=v / v_{\mathrm{NGS}}$. Assuming that the form of the AO rejection transfer function does not depend on aperture, we see that $\sigma_{\text {LGS }}^{2} \propto D^{4} \gamma_{\text {NGS }}^{\beta+1} \propto D^{6+2 \beta}$. For our full data set, the mean value of $\beta$ is -1.87 , so the RMS focus error due to sodium altitude variations increases typically in proportion to $D^{1.13}$.

In our measurements, system noise, from laser mode instability and photon shot noise, becomes dominant at frequencies above a few Hz. Rocket measurements (Chandra et al. 2008) indicate that the inner scale of turbulence in the mesosphere is of order $10 \mathrm{~m}$. With a typical average wind speed of $\sim 30 \mathrm{~ms}^{-1}$, we would thus expect the PSD to turn down above a few Hz. In any case, the power law is not expected to continue much above $\sim 30 \mathrm{~Hz}$. This frequency corresponds to the advection of spatial scales of $\sim 1 \mathrm{~m}$, the mean free path at the sodium altitude. Molecular viscosity will attenuate fluctuations on smaller scales.

Long-term variations in the mean values of $\alpha$ and $\beta$ are seen in Fig. 11 that are well outside the standard errors for these quantities. The entire three-year data set was analyzed using the same software and hence systematics from the analysis can be excluded. The variability thus likely arises from long-term weather patterns. Variations in the amplitude $\alpha$ have the greatest impact on AO performance. Yearly averages of this parameter can vary by a factor of two to three, which will be reflected in the contribution of sodium variability to the $\mathrm{AO}$ error budget. A preliminary investigation shows correlation between the normalization of the temporal mean altitude power spectrum an the southern oscillation index, an indicator derived from pressure differences in the southern Pacific, also showing the El Nino effect. Further work is needed to explore this effect.

While the temporal mean altitude power spectrum shows indices around -1.9 , the vertical spatial power spectrum shows indices varying mostly between -2 and -3 . The index of a spatial power spectrum indicates the influence of gravity wave interaction compared to intrinsic turbulent motion due to stratified shear flow. Spatial power spectrum indices typically vary between -2 and -3.5 (Beatty et al. 1992; Senft et al. 1993; Hines 1991), which are significantly steeper than the Kolmogorov index of $-5 / 3$ indicating a predominace of processes other than turbulence.

\section{Summary and conclusions}

This paper summarizes results from a three-year campaign of observations using the LZT Lidar Facility. Table 2 presents a summary of the most-relevant statistical parameters.

The sodium width was determined using the enclosed energy parameter. This was done with and without binning to simulate $0.5^{\prime \prime}$ wavefront sensor CCD pixels. While the enclosed energy parameter gave about the same width on average, the $90 \%$ of
Table 2. Main results from the three-year data set.

\begin{tabular}{|c|c|c|c|c|}
\hline $\begin{array}{l}\text { Sodium } \\
\text { layer } \\
\text { parameter }\end{array}$ & $\begin{array}{c}\text { Mean } \\
\text { all } \\
\text { data }\end{array}$ & 2008 & 2009 & 2010 \\
\hline $\begin{array}{l}\text { Centroid } \\
\text { altitude } \\
{[\mathrm{km}]}\end{array}$ & $\begin{array}{l}90.8 \\
\pm 0.1\end{array}$ & $\begin{array}{l}90.5 \\
\pm 0.1\end{array}$ & $\begin{array}{l}90.7 \\
\pm 0.2\end{array}$ & $\begin{array}{l}91.1 \\
\pm 0.1\end{array}$ \\
\hline $\begin{array}{l}\text { Width }(95 \% \\
\text { ePC) } \\
{[\mathrm{km}]}\end{array}$ & $\begin{array}{l}13.1 \\
\pm 0.3\end{array}$ & $\begin{array}{l}14.5 \\
\pm 0.5\end{array}$ & $\begin{array}{l}11.6 \\
\pm 0.5\end{array}$ & $\begin{array}{l}13.3 \\
\pm 0.4\end{array}$ \\
\hline $\begin{array}{l}\text { Width }\left(0.5^{\prime \prime}\right. \\
\text { px-95\% } \\
\text { ePC) }[\mathrm{km}]\end{array}$ & $\begin{array}{r}13.3 \\
\pm 0.2\end{array}$ & $\begin{array}{l}13.2 \\
\pm 0.5\end{array}$ & $\begin{array}{l}13.8 \\
\pm 0.5\end{array}$ & $\begin{array}{l}13.1 \\
\pm 0.3\end{array}$ \\
\hline $\begin{array}{l}\text { Temp. PS } \\
\text { Norm. } \\
{\left[\mathrm{m}^{2} / \mathrm{Hz}\right]}\end{array}$ & $\begin{array}{l}34.4 \\
+5.6 \\
-4.8\end{array}$ & $\begin{array}{c}28.5 \\
+7.6 \\
-6.0\end{array}$ & $\begin{array}{l}19.4 \\
+4.8 \\
-3.9\end{array}$ & $\begin{array}{l}51.5 \\
+13.9 \\
-11.0\end{array}$ \\
\hline $\begin{array}{l}\text { Temp. PS } \\
\text { Slope [-] }\end{array}$ & $\begin{array}{l}-1.87 \\
\pm 0.02\end{array}$ & $\begin{array}{l}-1.93 \\
\pm 0.03\end{array}$ & $\begin{array}{l}-1.97 \\
\pm 0.04\end{array}$ & $\begin{array}{l}-1.78 \\
\pm 0.03\end{array}$ \\
\hline $\begin{array}{l}\text { Non-photon } \\
\text { noise [\%] }\end{array}$ & $\begin{array}{r}22.4 \\
\pm 1.0 \\
\end{array}$ & $\begin{array}{r}12.9 \\
\pm 1.0 \\
\end{array}$ & $\begin{array}{r}23.8 \\
\pm 1.2 \\
\end{array}$ & $\begin{array}{r}26.5 \\
\pm 1.5 \\
\end{array}$ \\
\hline
\end{tabular}

Notes. "ePC" means enclosed photon counts. Non-photon noise refers to the difference between the variance due to photon noise and the flat white noise spectrum, fitted for the temporal power spectrum.

peak flux parameter gave sodium widths much higher for the binned case.

The power spectrum analysis results in three important parameters, the slope and normalization, important for AO end-toend simulations. The third parameter is the noise, which shows up as a flattening of the power spectrum at high frequencies. As Table 2 shows, the measured noise is somewhat larger than the photon noise calculated from Poisson statistics. The additional noise might arise from transparency fluctuations if thin cirrus clouds are present. Or there may be other sources of noise in the system, such as electron multiplication noise in the photomultipliers. Environmental influences such as low or high external temperatures might conceivably have an impact on the performance. Our data were obtained at ambient temperatures ranging from $-14^{\circ} \mathrm{C}$ to $+30^{\circ} \mathrm{C}$.

Meteor trails were often detected so their rates and effects on the mean altitude were determined. Most meteor trails are weak enough to not alter the mean altitude significantly. There are however a few significant meteors that can change the mean altitude by as much as $1 \mathrm{~km}$ in less than a second. Such events have to be considered when designing AO control algorithms. The average rate for these significant meteors is approximately 0.2 per hour.

Horizontal structure in the sodium is important for AO systems employing multiple LGS. If we assume that Taylor's frozen flow hypothesis is valid in the mesosphere, the horizontal mean altitude structure function, in the direction of the mean wind, can be estimated from the temporal power spectrum Pfrommer $\&$ Hickson (2010). However, the frozen flow assumption is questionable in this region due to the predominance of gravity waves. Therefore direct measurements are essential. To address this question, we have begun a program of horizontal sodium structure measurements with the LZT lidar system. The results will be presented in a future paper. 
T. Pfrommer and P. Hickson: High resolution mesospheric sodium properties for adaptive optics applications

Acknowledgements. This work was supported by the Thirty Meter Telescope Project and the European Southern Observatory. The LZT lidar system was constructed with funds from the Natural Sciences Engineering Research Council of Canada (NSERC), the Canada Foundation of Innovation, and the British Columbia Knowledge Development Fund. We thank R. Holzlöhner, D. Bonaccini-Calia, W. Hackenberg and S. Lewis for valuable advice in portions of this work. P.H. thanks the European Southern Observatory and the Université de Liège for hospitality and support during sabbatical visits, where a portion of this work was completed. Financial support to P.H. was provided by an NSERC Discovery Grant. We are indebted to Thierry Forveille for pointing out that a higher temporal sampling frequency, possible with larger telescope apertures, will at least partly mitigate the aperture dependence of focus error on sodium altitude variations.

\section{References}

Beatty, T. J., Hostetler, C. A., \& Gardner, C. S. 1992, J. Atmos. Sci., 49, 477 Beckers, J. M. 1993, Ann. Rev. Astron. Astrophys., 31, 13

Brown, P., Weryk, R. J., Wong, D. K., \& Jones, J. 2008, Icarus, 195, 317

Brown, P., Wong, D. K., Weryk, R. J., \& Wiegert, P. 2010, Icarus, 207, 66

Ceplecha, Z., Borovička, J., Elford, W. G., et al. 1998, Space Sci. Rev., 84, 327

Chandra, H., Sinha, H. S. S., Das, U., et al. 2008, Ann. Geophys., 26, 2725

Clemesha, B. R., Kirchhoff, V. W. J. H., Simonich, D. M., Takahashi, H., \& Batista, P. P. 1980, J. Geophys. Res., 85, 3480

Conan, R., Lardière, O., Herriot, G., Bradley, C., \& Jackson, K. 2009, Appl. Opt., 48, 1198

Davis, D. S., Hickson, P., Herriot, G., \& She, C.-Y. 2006, Opt. Lett., 31, 3369

Ellerbroek, B., Adkins, S., Andersen, D., et al. 2008, SPIE Conf. Ser., 7015

Gardner, C. S., Voelz, D. G., Sechrist, Jr., C. F., \& Segal, A. C. 1986,

J. Geophys. Res., 91, 13659
Herriot, G., Hickson, P., Ellerbroek, B., et al. 2006, in SPIE Conf. Ser., 6272

Hickson, P., Pfrommer, T., Cabanac, R., et al. 2007, PASP, 119, 444

Hines, C. O. 1991, J. Atmos. Sci., 48, 1361

Holzlöhner, R., Rochester, S. M., Bonaccini Calia, D., et al. 2010, A\&A, 510, A20

Kane, T. J., \& Gardner, C. S. 1993, Science, 259, 1297

Liu, Y., \& Yi, F. 2009, J. Atmos. Sol.-Terr. Phys., 71, 1374

Lyubenova, M., \& Kissler-Patig, M. 2009, An Expanded View of the Universe Science with the European Extremely Large Telescope, Tech. Rep., European Southern Observatory

Neichel, B., D'Orgeville, C., Callingham, J., et al. 2013, MNRAS, 429, 3522

Pfrommer, T. 2010, Ph.D. Thesis, University of British Columbia

Pfrommer, T., \& Hickson, P. 2010, J. Opt. Soc. Am. A, 27, 97

Pfrommer, T., Hickson, P., She, C., \& Vance, J. D. 2008, in SPIE Conf. Ser., 7015

Pfrommer, T., Hickson, P., \& She, C.-Y. 2009, Geophys. Res. Lett., 36, 15831

Plane, J. M. C. 2003, Chem. Rev., 103, 4963

Senft, D. C., Hostetler, C. A., \& Gardner, C. S. 1993, J. Atmos. Terr. Phys., 55, 425

She, C. Y., Yu, J. R., Huang, J. W., Nagasawa, C., \& Gardner, C. S. 1991, Geophys. Res. Lett., 18, 1329

Silva, D., Hickson, P., Steidel, C., \& Bolte, M. 2007, TMT Detailed Science Case (TMT Observatory Corporation)

von Zahn, U. 2005, in 17th ESA Symp. on European Rocket and Balloon Programmes and Related Research, ed. B. Warmbein, ESA SP, 590, 33

von Zahn, U., \& Hansen, T. L. 1988, J. Atmos. Terr. Phys., 50, 93

von Zahn, U., Gerding, M., Höffner, J., McNeil, W. J., \& Murad, E. 1999, Meteor. Planet. Sci., 34, 1017

Wang, L., Ellerbroek, B., \& Veran, J. P. 2009, Appl. Opt., 48, 5076

Yuan, T., She, C., Krueger, D. A., et al. 2008, J. Geophys. Res., 113, 3105 